Running Head: Mu suppression and empathic accuracy

\title{
Mu rhythm suppression over sensorimotor regions is associated with greater empathic accuracy
}

\author{
Shir Genzer ${ }^{*}$, Desmond C. Ong $2,3{ }^{*}$, Jamil Zaki ${ }^{4}$, and Anat Perry ${ }^{1}$ \\ ${ }^{1}$ Department of Psychology, Hebrew University of Jerusalem, Jerusalem, Israel \\ ${ }^{2}$ Department of Information Systems and Analytics, National University of Singapore \\ ${ }^{3}$ Institute of High Performance Computing, Agency for Science Technology and Research, \\ Singapore \\ ${ }^{4}$ Stanford University, Stanford, CA \\ *Denotes equal contribution
}

Manuscript dated: June 26, 2021

Address Correspondence to:

Anat Perry

Psychology Department

Hebrew University of Jerusalem

Jerusalem, Israel

anat.perry@mail.huji.ac.il

Word count: 7685 (excluding tables, legends, references)

Abstract word count: 200

Number of references: 57

Author Contributions: DCO, JZ, and AP designed the experiments. SG and AP collected the data, and DCO, SG, and AP analyzed the data. All authors wrote the paper.

The authors declare no competing interests and the data presented in this manuscript have not been used in prior published manuscripts. 


\begin{abstract}
When people encounter others' emotions, they engage multiple brain systems, including parts of the sensorimotor cortex associated with motor simulation. Simulation-related brain activity is commonly described as a "low-level" component of empathy and social cognition. It remains unclear whether and how sensorimotor simulation contributes to complex empathic judgments. Here we combine a naturalistic social paradigm with a reliable index of sensorimotor cortex-based simulation: EEG suppression of oscillatory activity in the mu frequency band. We recruited participants to watch naturalistic video clips of people ("targets") describing emotional life events. In two experiments, participants viewed these clips (i) with video and sound, (ii) with only video, or (iii) with only sound and provided continuous ratings of how they believed the target felt. We operationalized empathic accuracy as the correlation between participants' inferences and targets' self-report. In Experiment 1 (US sample), across all conditions, right-lateralized mu suppression tracked empathic accuracy. In Experiment 2 (Israeli sample), this replicated only when using individualized frequency-bands and only for the visual stimuli. Our results provide novel evidence that sensorimotor representations-as measured through mu suppression-play a role not only in low-level motor simulation, but also in higher-level inferences about others' emotions, especially when visual cues are crucial for accuracy.
\end{abstract}

Keywords: Empathic Accuracy; Mu Suppression; Inference; EEG; Affective Cognition 
Imagine that you are consoling a crying friend: Her emotional expressions, her tone of voice, and what she says all provide rich cues to how she feels, which you naturally piece together to understand her experience. Our inferences about each other are often quite accurate (Zaki \& Ochsner, 2011), though imperfect (Eyal et al., 2018).

Empathy is a multifaceted phenomenon (e.g., Decety \& Meyer, 2008; Zaki, 2014), and people can draw on multiple empathic processes when evaluating others' emotional and mental states. One such process is experience sharing, which refers to people's tendency to vicariously share the internal states of others (e.g., Levenson \& Ruef, 1992). Another is mentalizing, involves a reasoning component, by which people use their knowledge of the world (their intuitive theories of other people) to reason about others' emotions, intentions, beliefs, and behaviors (Ong et al., 2015; Saxe \& Houlihan, 2017). Experience sharing and mentalizing rely on dissociable systems of brain regions, and these processes are triggered preferentially by different classes of social cues (Van Overwalle \& Baetens, 2009; Zaki \& Ochsner, 2012). Brain regions engaged by mentalizing processes (such as the medial prefrontal cortex, mPFC) are preferentially activated by reading cues describing how others' emotional and mental states arise in context (e.g., Skerry \& Saxe, 2015). By contrast, experience sharing engages brain regions such as the anterior insula and anterior cingulate cortex for pain (e.g., Singer et al., 2004), or parietal and premotor regions for cues about others' sensorimotor states, such as photographs of facial expressions or motor actions (e.g., Keysers et al., 2010).

A well-established neural signature of experience sharing is mu suppression, measured via electroencephalography (EEG) or magnetoencephalography (MEG) (for a meta-analysis, see Fox et al., 2016). Neurons in the sensorimotor cortex tend to fire 
synchronously at rest, resulting in oscillations in the range of $8-13 \mathrm{~Hz}$, often termed mu rhythms. Suppression of these mu rhythms, resulting from increased sensorimotor activity (event-related desynchronization), occurs both when executing motor actions and when observing similar motor actions in others (Fox et al., 2016; Perry \& Bentin, 2009; Pineda, 2005). Previous studies have found increased mu suppression when viewing and making judgments about social stimuli, such as perceiving intentionality and emotions from motion (Perry, Troje, et al., 2010), viewing emotional facial expressions (Ensenberg et al., 2017; Moore et al., 2012; Popov et al., 2013; Rayson et al., 2016), viewing others' pain (Perry, Bentin, et al., 2010), playing a game with others (Perry et al., 2011), and making mentalstate attributions (Gutsell et al., 2020; Pineda \& Hecht, 2009). It is correlated with trait measures of empathic concern (DiGirolamo et al., 2019) and inversely correlated with dehumanization (Simon \& Gutsell, 2021).

Most previous studies examined the relationship between mu suppression and empathy through visual stimuli. However, in real life, when we interact with other people and try to understand them, we often not only see them but also hear them. Furthermore, in some cases, like a phone call, we can only hear the other. Therefore, it is essential to investigate if mu suppression is associated with empathy in general or if this association depends on the information presented in the stimulus. A few studies have investigated the role of mu suppression in processing social auditory stimuli (e.g., Hobson \& Bishop, 2017). However, these auditory tasks mainly focus on discriminating speech in noise (Cuellar et al., 2012; Jenson et al., 2014), or listening to language describing actions versus abstract concepts (e.g., Moreno et al., 2015). These results do not yet speak to how these processes contribute to the semantic understanding required for more complex empathic inferences. 
Therefore, in the current manuscript, we want to investigate not only the relationship between mu suppression and empathic inferences but also the influence of the information channels of the stimuli on this association.

The mounting evidence from the literature suggests that mu suppression is linked to inferences about others from low-level visual motor cues such as photographs, and possibly low-level auditory cues (see Simon \& Gutsell, 2021, for an exception). But the identification and discrimination tasks used in these previous experiments fall short of the complexity of everyday affective reasoning, and it is not clear whether or how these representations contribute to higher-level reasoning about others' affective states, especially in naturalistic contexts (e.g., Ong et al., 2015; Zaki et al., 2008). Thus, we designed the current study to test the hypothesis that motor representations of others' actions and expressions, as indexed by mu suppression, support people's ability to draw accurate inferences about others' affect in naturalistic contexts (Levenson \& Ruef, 1992; Zaki, Weber, et al., 2009).

In the first experiment (set in the U.S.), we adapted a task that we had previously used (Zaki, Bolger, et al., 2009) in which participants ("observers") watch videos of other people ("targets") recounting emotional autobiographical stories (Figure 1A). As observers are watching these videos, they provide continuous ratings of targets' affective state throughout the video. Targets' continuous ratings of their own affect were previously collected, enabling calculation of a measure of empathic accuracy for each observer watching each video (Zaki et al., 2008; Zaki, Bolger, et al., 2009; Zaki, Weber, et al., 2009). Observers in the current study were shown these autobiographical stories in three viewing conditions: They rated the targets' affect while watching a muted video (i.e., using only 
visual information, "Video-Only"); while only listening to the sound with no video (i.e., using only auditory information, "Audio-Only"); or while watching the video with audio (i.e., with both channels of information, "Audio-Video"). Similar to previous studies (Gesn \& Ickes, 1999; Hall \& Schmid Mast, 2007; Jospe et al., 2020), we hypothesized that observers will perceive another's affective state better than chance when having just the visual information, and significantly better when auditory (linguistic) information is present (Jospe et al., 2020; Zaki et al., 2009). Going beyond previous studies that looked only at identification and discrimination of simple social stimuli, we were interested in how mu suppression contributes to complex emotional inferences. Thus, insofar as mu suppression tracks processing of emotionally-relevant information across both visual and auditory modalities, we hypothesized that mu suppression should contribute to more accurate empathic judgments, across all conditions. Moreover, we hypothesized that the correlation between mu suppression and empathic accuracy will be the most substantial in the muted video-only condition. Finally, most prior studies examined mu suppression during short stimuli presentations (e.g., static photographs or 2-second-long video clips; see Fox et al., 2016, for a meta-analysis), so there is almost no evidence on the temporal dynamics of mu and the accuracy of social understanding. Naturalistic emotional understanding in particular fluctuates in small time intervals (Devlin et al., 2016; Zaki et al., 2008). Therefore, we wanted to test the hypothesis that mu suppression during shorter time intervals is related to more accurate affect judgments within that interval.

In the second experiment, we aimed to replicate the results of Experiment 1 using a larger sample size and a different stimuli set, in a different language and culture. Therefore, 
the procedures were identical to Experiment 1, except that Experiment 2 was set in Israel, using Israeli stimuli in Hebrew (Jospe et al., 2020).

\section{Experiment 1}

\section{Methods}

Participants. We recruited 21 English-speaking undergraduate students from the University of California, Berkeley, who received course credit for participating in the experiment. We excluded one participant from analysis due to technical problems with the EEG recordings, resulting in a final sample of 20 (18 female, mean age $=20.20$ years, $\mathrm{SD}=$ 2.30; information about handedness was not collected), from diverse ethnic backgrounds (1 American Indian, 8 East Asian, 1 Pacific Islander, 5 White, 5 Latin, 5 Southeast Asian, collected as self-reports from the participants and following the guidelines suggested by Flanagin et al., 2021). All participants reported normal or corrected to normal visual acuity and had no history of psychiatric or neurological disorders as confirmed by a screening interview.

Stimuli. We used videos collected as part of a previous project (Ong et al., 2019). Research volunteers (hereafter, "targets"; N = 68; 40 female, 26 male, 2 not reported; mean age 23.2 years) participated in exchange for monetary compensation and gave their informed consent as approved by the Stanford University Institutional Review Board. Targets were video-recorded narrating 3 positive and 3 negative autobiographical emotional events from their lives. After they finished recording these events, targets then watched their own videos and gave a continuous rating of how positive or negative they 
felt while speaking, using a 100-point rating slider (with endpoints "Very Negative" to "Very Positive"). The slider allowed targets to continuously update their affect ratings during the video ( see Ong et al., 2019, for more details on the stimuli recording and the targets rating procedure and Zaki et al., 2008; Jospe et al., 2020 for a similar approach). We selected 9 videos, all containing unique targets from this library. We chose stories that were comprehendible, with at least some facial expressions, and which did not include any names of people, and balanced the number of videos with mostly negative (4), mostly positive (3), and both negative and positive content (2), and the number of videos with male (4) and female (5) targets. For technical reasons, one of these videos did not have the target's continuous valence rating, so an empathic-accuracy score could not be computed for this video (see below). The length of the videos ranged between 1 minute 45 seconds and 3 minutes 24 seconds, with an average of 2 minutes 22 seconds. These 9 videos were then grouped into 3 between-subjects sets of equal duration (range: 425-431 seconds), such that participants in the present study saw a similar duration of audio-only, visualonly, or audiovisual stimuli. The assignment of these sets to condition (i.e., which videos were audio-only, visual-only, or audiovisual) was counterbalanced across participants.

Task. We used a modified version of an empathic-accuracy task that has been used before on several occasions (Devlin et al., 2016; Zaki et al., 2008; Zaki, Bolger, et al., 2009; Zaki, Weber, et al., 2009, see Figure 1A). In the first half of the session, we recorded EEG from participants while they passively viewed the 9 videos. Participants sat approximately $80 \mathrm{~cm}$ from the screen and were instructed to carefully notice how the target in the video feels at every moment in time, paying special attention to the momentary changes in the target's emotion. Each participant saw 3 video clips in the Audio-Video condition, 3 clips in 
the Video-Only condition, and 3 clips in the Audio-Only condition, in a randomized order (see Stimuli section above). All stimuli were preceded by a 5-second fixation point. We interspersed attention checks throughout the task (after every 2 or 3 videos), where participants had to answer a question about the video's content (e.g., "In the previous story, what was the mother diagnosed with?"). Note that the attention checks followed only the audio-only or audiovisual videos, as the visual-only videos had no semantic content.

Following this, participants viewed the same videos again, in the same order, without EEG. This time, participants provided continuous ratings of how positive or negative they thought the target felt while speaking, using a 100-point rating slider, from "Very Negative" to "Very Positive". This active rating portion was done outside the EEG setup to avoid contamination of the EEG signal-and especially of mu suppression — by the motor movement associated with making ratings. Note that the blocks' order (i.e., the EEG recording and continuous ratings) was fixed to ensure that the EEG signal would be most spontaneous and not be affected by habituation or prediction. Of course, the behavioral rating may have been affected by these (see discussion).

A

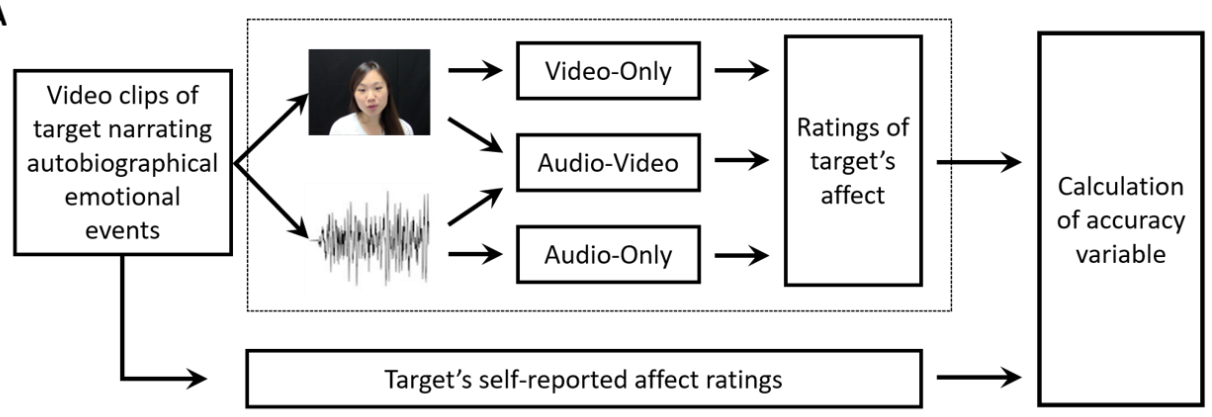

B

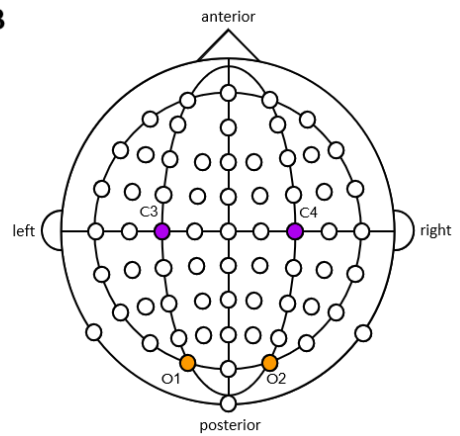

Figure 1. (A) Schematic of the task that participants performed (outlined in the dashed box). Participants are presented with multiple video clips in each of three viewing conditions: Video-Only (with no audio), Audio-Video, and Audio-Only (with no video). They provide continuous ratings of how they thought the target in the video felt. Together with the target's self-reported affect ratings, which we collected previously, we can calculate the accuracy variables that are our 
dependent measures. (B) Illustration of the four sites that were analyzed: C3 and C4 in the Central region, and 01 and 02 in the Occipital region.

EEG data acquisition. We recorded EEG continuously (from DC with a low-pass filter set at $100 \mathrm{~Hz}$ ) from $64 \mathrm{Ag}$-AgCl pin-type active electrodes mounted on a Biosemi elastic cap (http://www.biosemi.com/headcap.htm). Recording was done according to the extended 10-20 system. In addition, we recorded from two electrodes placed at the right and left mastoids. During recording, all electrodes were referenced to the common-mode signal electrode between POz and P03; they were subsequently re-referenced digitally (see Data Processing). To monitor eye movements and blinks, we measured bipolar horizontal and vertical Electrooculography (EOG) derivations using two electrode pairs. One pair was attached to the outer canthi of both eyes, while the other was attached to the infraorbital and supraorbital regions of the right eye. We digitally amplified, and sampled at $1024 \mathrm{~Hz}$, both EEG and EOG using a Biosemi Active II system (www.biosemi.com).

EEG data processing. We analysed the EEG data using the Brain Vision Analyzer software (Brain Products). We filtered the raw EEG data using a $0.5 \mathrm{~Hz}$ high-pass filter, a $30 \mathrm{~Hz}$ low-pass filter $(24 \mathrm{~dB})$, and a notch filter at $60 \mathrm{~Hz}$. Following filtering, the data were re-referenced offline to the average signal from the mastoid electrodes. We corrected EEG deflections resulting from eye movements and blinks using Interdependent Component Analysis (ICA; Jung et al., 2000), and we removed any remaining artifacts that exceeded plus minus 100 microvolts in amplitude. We segmented each video into 3 -second time windows ("epochs"), as previous mu studies have shown that mu suppression can be reliably estimated in these intervals (e.g., see Fox et al., 2016). We used the first 3-second epoch of each video to serve as a baseline for that video. For each epoch, we used a Fast 
Fourier Transform (FFT) at $0.5 \mathrm{~Hz}$ intervals and with a Hanning window to compute the integrated power in the $8-13 \mathrm{~Hz}$ range.

EEG measures. For our dependent variable, we defined a suppression index as the natural logarithm (ln) of the ratio of the power during each epoch relative to the power during the fixation period preceding that video (i.e., that video's baseline; e.g., Perry, Bentin, et al., 2010). We used the ratio of powers, as opposed to a simple subtraction, to control for the variability in absolute EEG power resulting from individual differences in scalp thickness and electrode impedance. In addition, the ratio data are inherently nonnormally distributed due to lower bounding, and so we applied a log transform. Greater mu suppression (i.e., less power compared to baseline) indicates more neuronal activation.

We computed suppression indices at four sites-C3 and 01 on the left hemisphere, and $\mathrm{C} 4$ and 02 on the right hemisphere-to compare suppression in the $8-13 \mathrm{~Hz}$ range between hemispheres and locations (Figure 1). We chose C3 and C4 as they are classic mu rhythm sites (Pineda, 2005), while the two occipital electrodes were chosen to contrast our predicted mu findings with occipital alpha suppression, a strong and well-known phenomenon attributed to visual-attentional mechanisms (Klimesch, 2012).

Behavioral measures. We had two dependent variables of interest. The first, what we term empathic accuracy following our earlier work (Zaki et al., 2008; Zaki, Bolger, et al., 2009), is a video-level summary of how accurately the participant judged the target's affect. Specifically, we operationalized this summary using the correlation of the participant's judgments with the target's own self-reported affect. As we intend to examine the correlation between the behavioral ratings and the EEG data, we adjusted the time scale of the behavioral data to match the time scale of the EEG data. Therefore, we 
segmented each video rating into 3-second intervals. The empathic-accuracy extracted measure is the correlation score between the participant's and the target's adjusted ratings.

Our second measure of accuracy, which we term change-detection accuracy, evaluates how accurately the participant assessed the target's emotional affect change. To operationalize this measure, we segmented each video rating into 3-second time windows ("epochs"). For each epoch, we classified participants' and targets' ratings into one of three categories: an increase in affect, a decrease, or maintained from the previous epoch. We then operationalized change detection such that a "successful" change detection occurred if a participant's rated change (i.e., increase, decrease, or maintain) matched the target's change at that epoch. If they did not match, this would be a "failed" change detection. Thus, change detection was a binary variable for each epoch that reflected whether the participant successfully detected any change (or lack thereof) in the target's affect (see Figure 2). Importantly, this definition is scale-invariant, in that it classifies changes without regard to the magnitude of the change, which helps to mitigate some issues with scale usage as it is not affected by how participants used the scale. Change detection is also "memory-less" such that ratings more than one epoch in the past do not affect this operationalization of accuracy, i.e., as opposed to a correlation, this calculation is not affected by the participant's accuracy before a given point, as each point is only relative to the one before it. 


\section{Calculation of the change-detection accuracy}
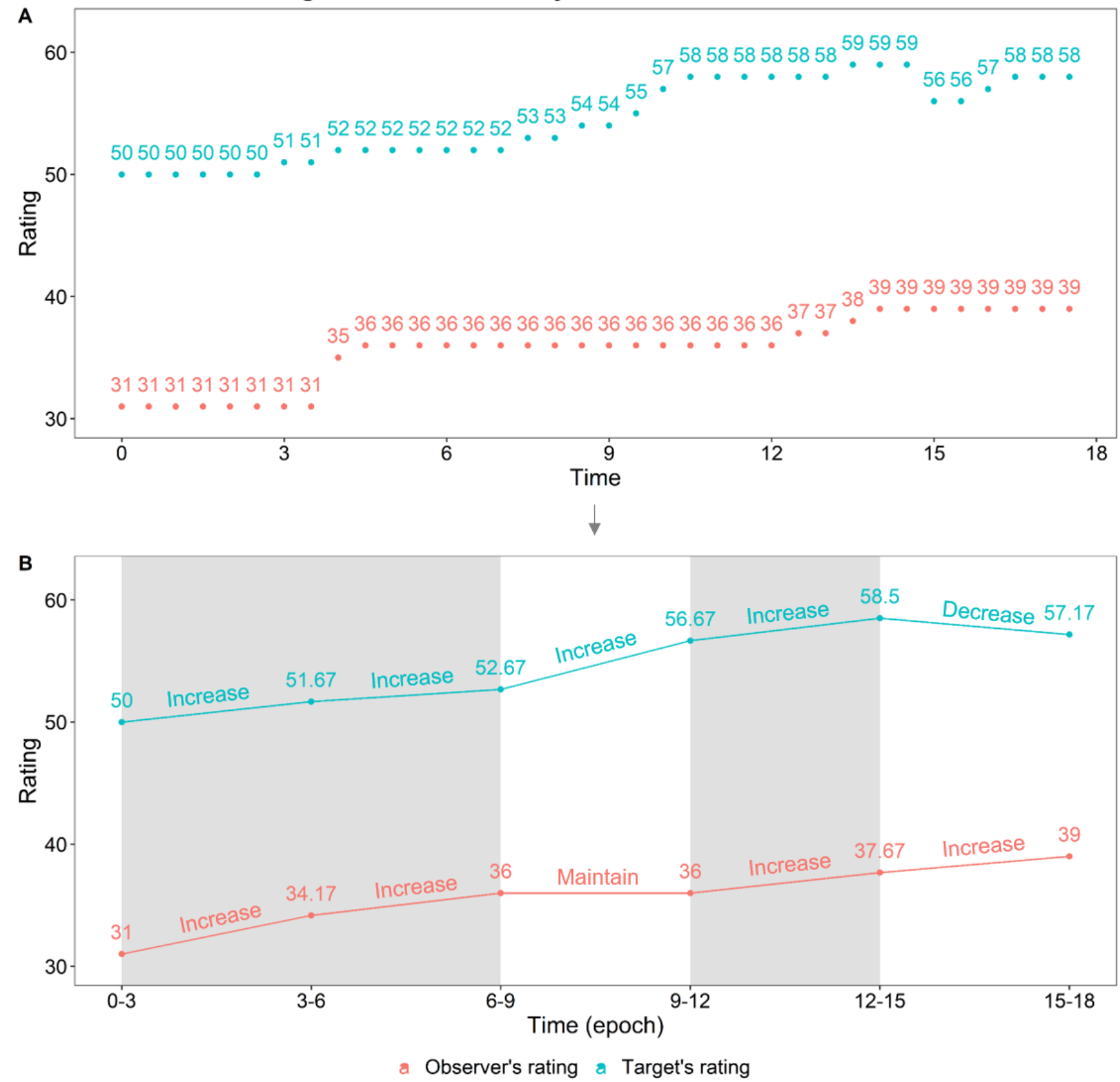

Figure 2. A visualization of the calculation stages of the change-detection accuracy score. (A) A sample of 27 seconds of target and observer ratings (every 0.5 second). (B) The epoch-level target and observer's ratings averaged for every 3 seconds and classification of ratings into one of three categories: an increase in affect, decrease, or maintained from the previous epoch. Shaded rectangles indicate epochs where the observer's rating change (increase, decrease, or maintain) matched the target's rating change. 
Statistical models. All statistical analyses were performed using R software (R Core Team, 2020). To ensure that the pattern of mu suppression in the central sites differed from those seen in the alpha occipital sites, we used two mixed-effects linear models to predict suppression over the central and the occipital sites with the lmer function from the Ime4 package (Bates et al., 2015). For the suppression measures, we averaged the suppression across the whole video, of the central site (averaged across C3 and C4), and the occipital site (averaged across 01 and 02). We added a categorical fixed effect for viewing condition (Audio-Video, Video-Only, and Audio-Only). We also added random effects by participant, and by video, to account for the crossed nature of the experiment's design: Each participant saw 9 videos, and each video was seen by all participants. To interpret the difference among all the viewing conditions, we conducted post-hoc contrasts with Bonferroni corrections (Audio-Video vs. Video-Only, Audio-Video vs. Audio-Only, and Audio-Only vs. Video-Only) using the emmeans function from the emmeans package (Lenth et al., 2020).

For the video-level analyses, we used a mixed-effects linear model to predict empathic accuracy using as our main predictor suppression, averaged across the whole video, from $\mathrm{C} 3, \mathrm{C} 4,01$, and 02 . We added a categorical fixed effect for viewing condition (Audio-Video, Video-Only, and Audio-Only). We also added random effects by participant, and by video, to account for the crossed nature of the experimental design. To test each variable's contribution to the model, we used a four-step hierarchical model approach. The first null model had no predictors and had only the random effects by participant and video. The suppression at the electrodes was added to the second model, the viewing condition was added to the third model, and the interaction between all the electrodes and 
the viewing condition was added to the fourth model. Then we compared the models with F-test estimations based on the Kenward-Roger approach using the function KRmodcomp from the pbkrtest package (Halekoh \& Højsgaard, 2014) to assess each variable's contribution to the model's goodness of fit. We interpreted the variable parameters from the most complex model, which significantly improved the model's goodness of fit, using the tab_model function from sjPlot package (Lüdecke, 2021); and we conducted post-hoc contrast comparisons with Bonferroni correction for the viewingcondition contrasts (Audio-Video vs. Video-Only, Audio-Video vs. Audio-Only, and AudioOnly vs. Video-Only).

As change detection is a binary variable (success/failure), we used a slightly different approach for the epoch-level analyses. Instead of using a linear mixed-effects model, we used a generalized linear mixed-effects model to predict change detection (i.e., a binomial variable) with the glmer function from the Ime4 package (Bates et al., 2015). Then we used a similar four-step hierarchical model approach with likelihood ratio test comparisons utilizing the anova function to assess each variable's contribution to the model's goodness of fit. Again, we interpreted the variables' parameters from the most complex model, which significantly improved the model's goodness of fit; and we conducted post-hoc contrast comparisons with Bonferroni correction for the viewingcondition contrasts (Audio-Video vs. Video-Only, Audio-Video vs. Audio-Only, and AudioOnly vs. Video-Only).

Note that all analyses were conducted on all data points without outlier removal to maintain as much statistical power as possible due to the small sample size. 
Data and code availability: Code for the behavioral analysis and statistical models can be found at: https://github.com/desmond-ong/EA-EEG

\section{Results}

First, we examined the levels of suppression across the different sites (Figure 3A). Over the central sites, participants exhibited the greatest mu suppression (less activation) while watching the Audio-Video clips, as compared to the Video-Only clips $(\beta=-0.14,95 \%$ Confidence Interval $[-0.19,-0.09], t=-5.77$, Bonferroni-corrected $p<0.001)$, and compared to the Audio-Only clips $(\beta=-0.15[-0.21,-0.10], t=-5.40, p<0.001)$. No difference was found between the Video-Only and Audio-Only conditions $(\beta=-0.01[-0.07,0.04], t=-0.37$, $p=1.00$ ). By contrast, over the occipital sites, participants exhibited the greatest alpha suppression (less activation) watching the silent Video-Only clips, as compared to watching the Audio-Video clips ( $\beta=-0.20[-0.25,-0.15], t=-7.96, p<0.001)$, and compared to listening to the Audio-Only clips $(\beta=-0.42[-0.48,-0.36], t=-14.81, p<0.001)$. There was also greater alpha suppression in the Audio-Video condition compared to the Audio-Only condition, which has no visual information $(\beta=-0.22[-0.28,-0.17], t=-7.85, p<0.001)$. The different patterns of mu and alpha suppression strengthen the notion that suppression over central sites reflects a different neural phenomenon that is reliant on both the visual and auditory modalities (Le Bel et al., 2009; Pineda, 2005).

Next, we turned to the video-level analyses predicting empathic accuracy. The model comparison indicated that suppression and condition, but not the interaction between them, significantly improved model goodness-of-fit (see Table 1 for model 
comparisons; for the full model see Table 2; reporting practices based on Aguinis et al., 2013).

\begin{tabular}{|c|c|c|c|c|c|}
\hline \multicolumn{2}{|c|}{ Model Comparison } & \multicolumn{2}{|c|}{$\begin{array}{l}\text { Video-Level Models: } \\
\text { Empathic Accuracy }\end{array}$} & \multicolumn{2}{|c|}{$\begin{array}{l}\text { Epoch-Level Models: } \\
\text { Change Detection }\end{array}$} \\
\hline Full model & Restricted model & $F$ statistic & $p$-value & $\chi^{2}$ statistic & $p$-value \\
\hline $\begin{array}{l}\text { Suppression } \\
\text { model }\end{array}$ & Null model & $F_{(4,126)}=4.34$ & 0.003 & $\chi_{(4)}^{2}=21.21$ & $<0.001$ \\
\hline $\begin{array}{l}\text { Suppression and } \\
\text { condition model }\end{array}$ & $\begin{array}{l}\text { Suppression } \\
\text { model }\end{array}$ & $F_{(2,112)}=16.51$ & $<0.001$ & $\chi_{(2)}^{2}=8.30$ & 0.02 \\
\hline $\begin{array}{l}\text { Interaction } \\
\text { model }\end{array}$ & $\begin{array}{l}\text { Suppression and } \\
\text { condition model }\end{array}$ & $F_{(8,114)}=0.76$ & 0.64 & $\chi_{(8)}^{2}=11.67$ & 0.17 \\
\hline
\end{tabular}

Table 1. Model comparison for Experiment 1, assessing the contribution of each variable (suppression at electrodes C3, $\mathrm{C} 4,01,02$; condition; and the interaction between them) to the goodness-of-fit of the (Left) linear mixed-effects model predicting participants' accuracy at rating the targets' affect across each video, and the (Right) generalized linear mixedeffects model predicting whether the participant's rating change at the epoch level (increased, decreased, or maintained, compared to the previous epoch) matched the target's rating change.

When we consider the main effects of condition in the suppression and condition model, there is higher empathic accuracy for the Audio-Video condition than the VideoOnly condition $(\beta=0.86[0.56,1.16], t=5.67$, Bonferroni-corrected $p<0.001)$. Higher empathic accuracy was also found in the Audio-Only condition compared to the Video-Only condition $(\beta=0.66[0.29,1.02], t=-3.56, p=0.002)$. No difference was found in empathic accuracy between Audio-Video and Audio-Only conditions ( $\beta=0.20[-0.13,0.54], t=1.17, p$ $=0.73$; Figure 3B; see Jospe et al., 2020, for similar findings). We then considered the main effects of suppression. Greater mu suppression in C4 (i.e., less activation of mu rhythms over the right sensorimotor cortex) was significantly associated with greater empathic accuracy $(\beta=-0.21[-0.40,-0.01], t=-2.04, p=0.04$; see Figure 3C). No significant 
correlation was found between mu suppression in C3 and empathic accuracy, or between alpha suppression in 01 and 02 and empathic accuracy (see Table 2). 


\begin{tabular}{|c|c|c|c|c|c|c|c|c|}
\hline \multirow[b]{2}{*}{ Predictors } & \multicolumn{4}{|c|}{$\begin{array}{l}\text { Video-Level Models: } \\
\text { Empathic Accuracy }\end{array}$} & \multicolumn{4}{|c|}{$\begin{array}{l}\text { Epoch-Level Models: } \\
\text { Change Detection }\end{array}$} \\
\hline & $\beta$ & $S E$ & $C I$ & $p$ & $\beta$ & $S E$ & $C I$ & $p$ \\
\hline Intercept & 0.33 & 0.21 & $-0.08,0.74$ & $<0.001$ & -0.32 & 0.10 & $-0.51,-0.12$ & 0.002 \\
\hline C3 suppression & 0.04 & 0.10 & $-0.17,0.24$ & 0.73 & -0.03 & 0.04 & $-0.11,0.05$ & 0.47 \\
\hline C4 suppression & -0.21 & 0.10 & $-0.40,-0.01$ & 0.04 & -0.12 & 0.04 & $-0.20,-0.04$ & 0.004 \\
\hline 01 suppression & 0.26 & 0.14 & $-0.01,0.53$ & 0.06 & 0.00 & 0.05 & $-0.10,0.10$ & 0.98 \\
\hline 02 suppression & -0.20 & 0.14 & $-0.49,0.08$ & 0.16 & 0.09 & 0.05 & $-0.02,0.19$ & 0.10 \\
\hline $\begin{array}{l}\text { Video-Only vs. } \\
\text { Audio-Video }\end{array}$ & -0.86 & 0.15 & $-1.16,-0.56$ & $<0.001$ & -0.17 & 0.06 & $-0.30,-0.05$ & 0.005 \\
\hline $\begin{array}{l}\text { Audio-Only vs. } \\
\text { Audio-Video }\end{array}$ & -0.20 & 0.17 & $-0.54,0.13$ & 0.24 & -0.03 & 0.07 & $-0.17,0.11$ & 0.65 \\
\hline \multicolumn{9}{|l|}{ Random Effects } \\
\hline$\sigma^{2}$ & \multicolumn{4}{|l|}{0.08} & \multicolumn{4}{|c|}{3.29} \\
\hline \multirow[t]{2}{*}{$\tau_{00}$} & \multicolumn{4}{|c|}{0.00 participantID } & \multicolumn{4}{|c|}{0.09 participantID } \\
\hline & \multicolumn{4}{|c|}{0.04 videolD } & \multicolumn{4}{|c|}{0.03 videoID } \\
\hline ICC & \multicolumn{4}{|l|}{0.35} & \multicolumn{4}{|c|}{0.03} \\
\hline \multirow[t]{2}{*}{$\mathrm{N}$} & \multicolumn{4}{|c|}{20 participantID } & \multicolumn{4}{|c|}{20 participantID } \\
\hline & \multicolumn{4}{|c|}{8 videoID } & \multicolumn{4}{|c|}{8 videoID } \\
\hline Observations & \multicolumn{4}{|l|}{137} & \multicolumn{4}{|c|}{6370} \\
\hline $\begin{array}{l}\text { Marginal R² / } \\
\text { Conditional R² }\end{array}$ & \multicolumn{4}{|c|}{$0.22 / 0.49$} & \multicolumn{4}{|c|}{$0.01 / 0.04$} \\
\hline AIC & \multicolumn{4}{|c|}{108.25} & \multicolumn{4}{|c|}{8485.02} \\
\hline Log-Likelihood & \multicolumn{4}{|c|}{-44.13} & \multicolumn{4}{|c|}{-4233.51} \\
\hline
\end{tabular}

Table 2. Summary of statistical models for Experiment 1. Left: Results from a linear mixed-effects model predicting participants' accuracy at rating the targets' affect across each video. Right: Results from a generalized linear mixed-effects model predicting whether participants' rating change at the epoch level (increased, decreased, or maintained, compared to the previous epoch) matched the targets' rating change. See Methods for operationalization of the dependent variables. $\beta$ indicates the standardized beta coefficients on suppression at the electrodes, where negative values indicate greater 
suppression. Note: SE: standard error of the regressor. CI: confidence intervals of the standardized beta coefficients of the regressor.

\section{A}

\section{Alpha and mu rhythms in the} different conditions

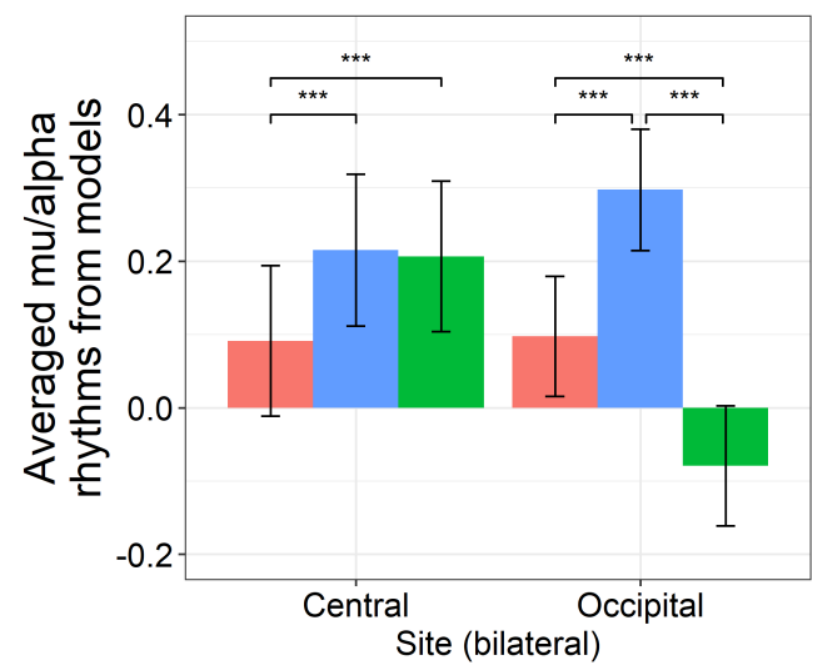

C

\section{Correlation between mu across conditions and empathic accuracy}

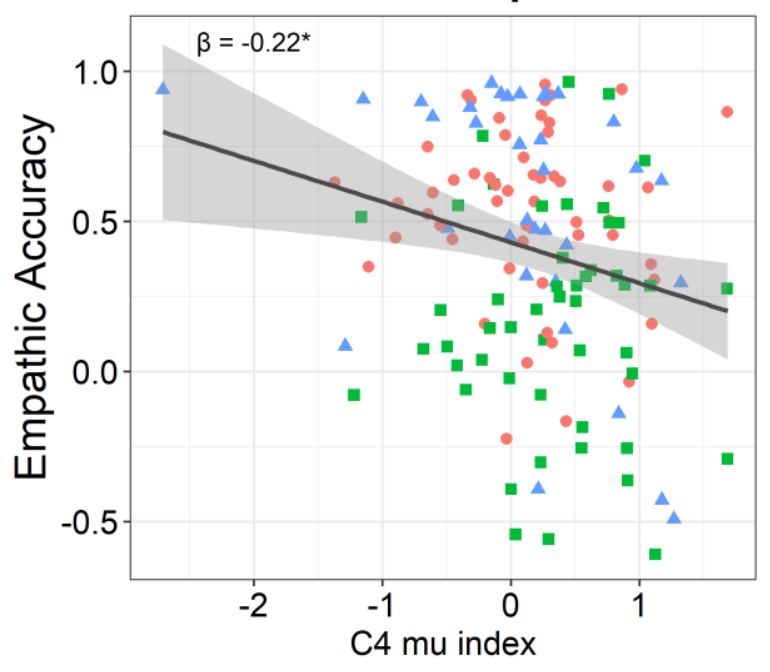

B Empathic Accuracy

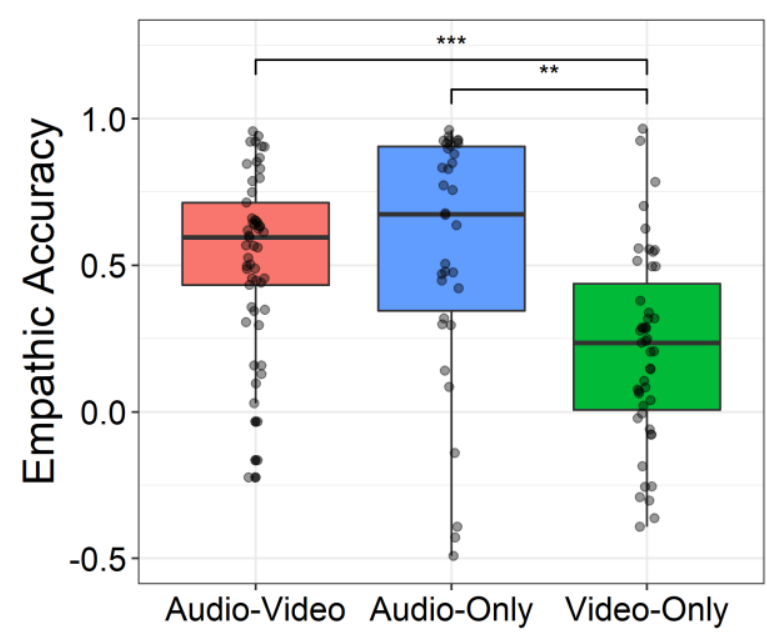

Figure 3. Experiment 1 results. (A) A barplot of mu and alpha rhythms in the different conditions over the central and occipital sites. Values obtained from the linear mixed-effects models predicting participants' mu and alpha rhythms in the different conditions. More negative values indicate more suppression relative to baseline. The error bars reflect SE. (B) A boxplot of empathic-accuracy scores in the different conditions. Values obtained from the linear mixed-effects models predicting participants' accuracy at rating the targets' affect across each video. (C) A scatterplot of video-level empathic accuracy against averaged mu suppression in electrode C4. Data-points are colored by viewing condition. On the horizontal axis, more negative values indicate greater suppression. The line represents a best-fit line, showing a negative 
correlation between mu suppression and accuracy. There was no interaction of suppression by condition on accuracy. ${ }^{*} p<.05,{ }^{* *} p<.01,{ }^{* * *}, p<.001$

Finally, we considered the epoch-level analyses predicting change detection. Model comparison similarly showed that suppression and condition, but not the interaction between them, significantly improved model goodness-of-fit (see Table 1 for model comparisons; for the full model see Table 2; reporting practices based on Aguinis et al., 2013; Nakagawa \& Schielzeth, 2013). Similar to the video-level model, this model revealed enhanced change detection for the Audio-Video condition compared to the Video-Only condition $(\beta=0.17[0.05,0.30], t=2.79$, Bonferroni-corrected $p=0.02)$. However, no significant difference was found between the Audio-Video and Audio-Only conditions $(\beta=$ $0.03[-0.11,0.17], t=0.46, p=1.00$ ), or between the Audio-Only and Video-Only conditions $(\beta=0.14[-0.003,0.29], t=-1.92, p=0.16)$. When we considered the main effects of suppression, consistent with the results for the video-level model, the epoch-level model also indicated that greater mu suppression in C4 (i.e., less activation of mu rhythms over the right sensorimotor cortex) was significantly associated with enhanced change detection $(\beta=-0.12[-0.20,-0.04], t=-2.87, p=0.004$; see Figure 4). No significant correlation was found between mu suppression in C3 and change detection, or between alpha suppression in 01 and 02 and change detection (see Table 2). 

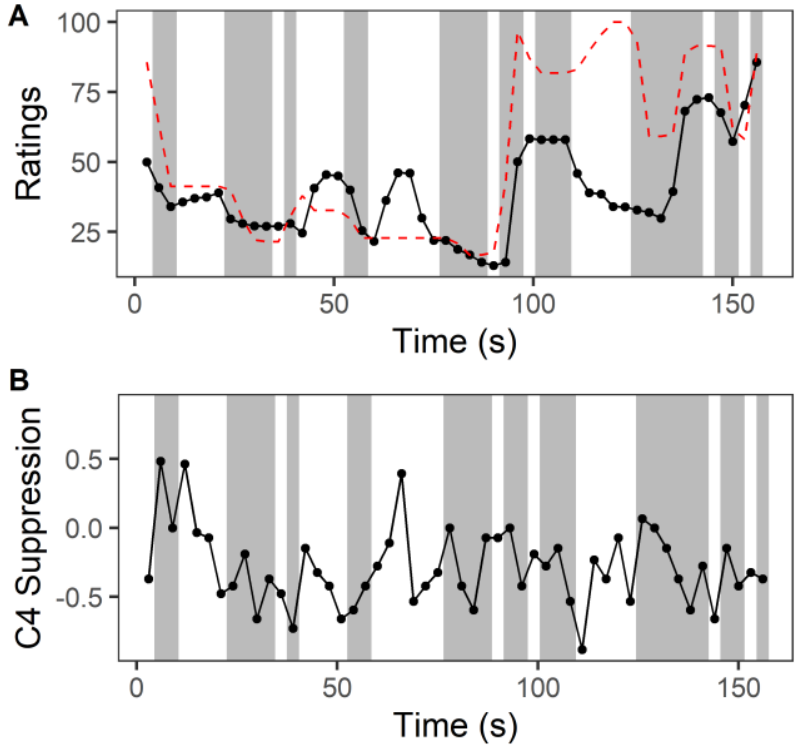

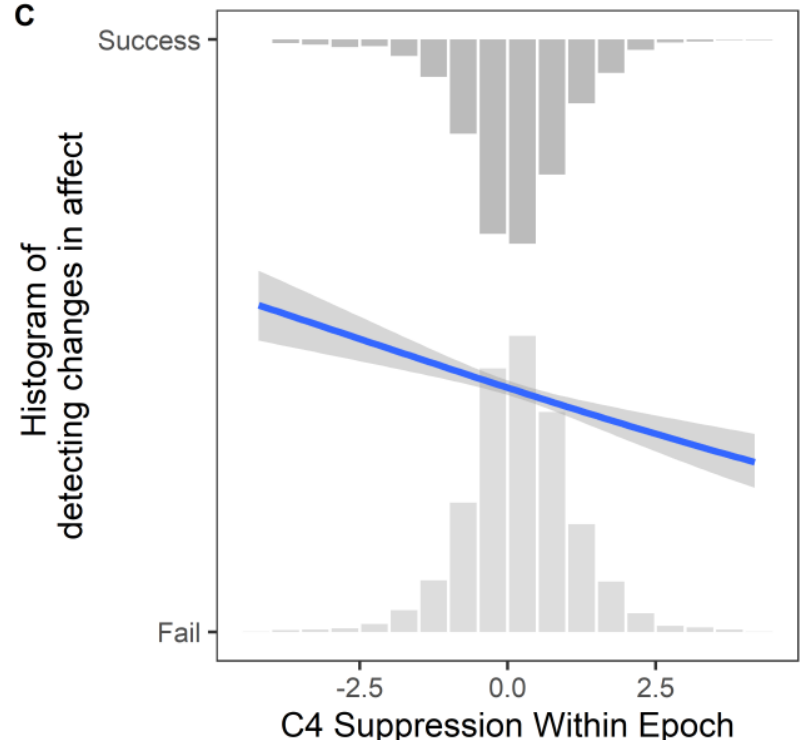

Figure 4. Examining change detection in Experiment 1. (A) Ratings made by a sample participant watching a clip in the Audio-Video condition, compared to the target's ratings (dashed red line). Ratings range from "Very Negative" (0) to "Very Positive" (100). Shaded rectangles indicate epochs where the participants' rating change (increase, decrease, or maintain) matched the target's rating change. (B) Corresponding suppression index in C4 for the same participant watching the same video clip. More negative values indicate more suppression. (C) This plot, averaged across all participants, shows the histogram of mu suppression in C4 for successful change detections (top histogram) and unsuccessful change detections (bottom histogram). More negative values on the horizontal axis indicate more suppression. The blue line represents a best-fit line from a binomial general linear model, showing a significant negative correlation: More C4 suppression is associated with a greater probability of successfully detecting a change in the target's affect.

\section{Discussion}

In Experiment 1 we found that greater mu suppression in electrode C4, over the right sensorimotor cortex, was significantly associated with greater empathic accuracy. This is in line with previous studies that found evidence for right-lateralized mu suppression involving recognition of emotional expressions (Moore et al., 2012; Rayson et al., 2016). Importantly, our results not only provide corroborating evidence that sensorimotor representations-specifically in the right hemisphere-are important in processing emotional information, they also reveal that these sensorimotor 
representations contribute to accurate affect judgments in complex naturalistic stories.

We did not find an interaction with condition, suggesting that these sensorimotor representations may not be limited to the visual modality and contribute to empathic accuracy across both visual and auditory modalities.

We also found that greater mu suppression over the right sensorimotor cortex (electrode C4) was significantly associated with greater accuracy at detecting epoch-toepoch changes in affect. To the best of our knowledge, this is the first time that mu suppression has been analyzed at such a fine-grained level within a stimulus, revealing sensorimotor sensitivity to changes in complex stimuli on a several-seconds-level resolution. These results, although correlational, strengthen the notion that the sensorimotor cortex may significantly add to understanding complex affective cues as they unfold in a natural environment.

\section{Experiment 2}

Due to the novelty of the previous experiment's findings, we conducted a second experiment to replicate the results. We used a larger sample size, and a different stimulus set, in a different language and culture, to further generalize the results. Experiment 2 was identical to Experiment 1, but it was conducted in Israel using an Israeli stimuli set, and stories were in Hebrew (Jospe et al., 2020).

\section{Methods}

Participants. We recruited 56 Hebrew-speaking undergraduate students from the Hebrew University of Jerusalem, who received either course credit or monetary 
compensation at a rate of 40 NIS per hour $(\sim 15)$ for participating in the experiment. We excluded six participants from analysis due to technical problems during the recordings and four participants due to massive EEG alpha waves, resulting in a final sample of 46 participants ( 25 female, mean age $=23.68$ years, $\mathrm{SD}=2.05,43$ right-handed). Information about ethnicity was not collected). All participants reported normal or corrected to normal visual acuity and had no history of psychiatric or neurological disorders, as confirmed by a screening interview.

Stimuli. We used videos in Hebrew from an Israeli empathic-accuracy stimuli set we collected as part of a previous project, in an identical manner to that described above (for full details, see Jospe et al., 2020). From this set, we selected 9 videos, all containing unique targets. We chose stories that were comprehendible, with at least some facial expressions, and which did not include any names of people and balanced the number of male (4) and female (5) targets, with mostly negative (4), mostly positive (3), and both negative and positive content (2). The lengths of the videos were between 2 minutes 2 seconds and 3 minutes 48 seconds, with an average of 2 minutes 43 seconds. These 9 videos were then grouped into 3 between-subjects sets of equal duration (range: 454-520 seconds), such that participants in the present study saw a similar duration of audio-only, visual-only, or audiovisual stimuli. The assignment of these sets to condition (i.e., which videos were audio-only, visual-only, or audiovisual) was counterbalanced across participants.

Task. The empathic-accuracy task was identical to Experiment 1, except for the different stimuli set.

EEG data acquisition. Identical to Experiment 1. 
EEG data processing. We conducted two EEG data-processing procedures: The first was identical to the one in Experiment 1 as a replication. It should be noted that although we generally define mu rhythms as oscillations in the range of $8-13 \mathrm{~Hz}$, the exact numerical boundaries of the mu frequency range are somewhat subjective, are variously defined in the literature as 7-12 Hz, 8-13 Hz, 9-11 Hz (Cohen, 2021) and may be affected by individual differences (Chiang et al., 2011). Therefore, we conducted an exploratory analysis following the initial analysis, in which we extracted individualized $2 \mathrm{~Hz}$ frequency bands of mu rhythm for each participant. This method may be more robust for finding effects, instead of a more smeared response that uses the general 8-13Hz range, (see Lepage \& Théoret, 2006; Muthukumaraswamy \& Johnson, 2004, for similar analyses). Individual mu rhythm bands were defined by the following procedure: For each participant and each video, we averaged the epoched data following the FFT procedure. Then, we averaged this data across videos to compute the average integrated power in the $8-13 \mathrm{~Hz}$ range for that participant across conditions. We manually identified the maximum power peak in the $8-13 \mathrm{~Hz}$ range and defined the $2 \mathrm{~Hz}$ frequency band adequacy $(1 \mathrm{~Hz}$ above and below the maximum power peak). If the maximum power peak could not be identified, we used $10 \mathrm{~Hz}$ as a default, and chose $9-11 \mathrm{~Hz}$ accordingly. Then, for each participant in each condition, we exported the FFT of that $2 \mathrm{~Hz}$ range, at $0.5 \mathrm{~Hz}$ intervals and with a Hanning window, to compute the integrated power of the individualized mu rhythm.

EEG measures. For our dependent variable, we defined a suppression in four sites $(\mathrm{C} 3, \mathrm{C} 4,01,02)$ as in Experiment 1, for both the full range and individualized EEG exported data. 
Behavioral measures. We extracted two dependent variables, the empathic accuracy score and the change-detection accuracy, as in Experiment 1.

Statistical models. Similar to Experiment 1 with two adjustments: (1) For the behavioral measures, we removed trials with 2 standard deviations away from the overall global mean empathic accuracy (for a similar procedure, see Jospe et al., 2020), which removed 29 data-points out of 402 (7.2\%); (2) Following the results of Experiment 1, and in order to increase the statistical power, for the four models' comparison, the last model with the interaction included interaction between viewing condition and electrode C4 only and not with all electrodes.

\section{Results}

Using the whole $8-13 \mathrm{~Hz}$ frequency range did not replicate the findings of Experiment 1 (for the analysis, see supplementary materials) - that is, there was no significant correlation between C4 mu suppression and empathic accuracy across the 8$13 \mathrm{~Hz}$ range.

We next conducted an exploratory analysis using the individual $2 \mathrm{~Hz}$ frequency range for each participant (see the Methods section), which may be more sensitive due to individual differences in peak frequencies (Lepage \& Théoret, 2006; Muthukumaraswamy \& Johnson, 2004). Here, similar to Experiment 1, the mixed-effects linear model across the central sites reflected a different suppression pattern compared to the occipital sites (Figure 5A). Over the central sites, we found no difference in mu suppression between the Video-Only and Audio-Video conditions $(\beta=-0.02[-0.05,0.01], t=-1.29$, Bonferronicorrected $p=0.59)$. However, greater suppression was found for the Video-Only condition 
compared to the Audio-Only condition $(\beta=-0.17[-0.20,-0.14], t=-11.47, p<0.001)$. There was also greater mu suppression in the Audio-Video than the Audio-Only condition $(\beta=-$ $0.15[-0.18,-0.12], t=-10.27, p<0.001)$. By contrast, over the occipital sites, participants exhibited greater alpha suppression when watching the silent Video-Only clips, as compared to when watching the Audio-Video clips $(\beta=-0.12[-0.15,-0.10], t=-8.72, p<$ $0.001)$ and compared to listening to the Audio-Only clips $(\beta=-0.42[-0.44,-0.39], t=-$ 28.91, $p<0.001$ ). There was also greater alpha suppression in the Audio-Video condition compared to the Audio-Only condition $(\beta=-0.29[-0.32,-0.26], t=-20.43, p<0.001)$.

Next, we turned to the model comparisons predicting empathic accuracy. The model comparisons suggest that suppression, condition, and-unlike Experiment 1 - the interaction between condition and mu suppression at C4 significantly improved the model goodness-of-fit (see Table 3; for the full model see Table 4).

\begin{tabular}{|c|c|c|c|c|c|}
\hline \multicolumn{2}{|c|}{ Model Comparison } & \multicolumn{2}{|c|}{$\begin{array}{l}\text { Video-Level Models: } \\
\text { Empathic Accuracy }\end{array}$} & \multicolumn{2}{|c|}{$\begin{array}{l}\text { Epoch-Level Models: } \\
\text { Change Detection }\end{array}$} \\
\hline Full model & Restricted model & $F$ statistic & $p$-value & $\chi^{2}$ statistic & $p$-value \\
\hline $\begin{array}{l}\text { Suppression } \\
\text { model }\end{array}$ & Null model & $F_{(4,247)}=1.55$ & 0.19 & $\chi_{(4)}^{2}=11.24$ & 0.02 \\
\hline $\begin{array}{l}\text { Suppression and } \\
\text { condition model }\end{array}$ & $\begin{array}{l}\text { Suppression } \\
\text { model }\end{array}$ & $F_{(2,330)}=91.62$ & $<0.001$ & $\chi_{(2)}^{2}=64.47$ & $<0.001$ \\
\hline $\begin{array}{l}\text { Interaction } \\
\text { model }\end{array}$ & $\begin{array}{l}\text { Suppression and } \\
\text { condition model }\end{array}$ & $F_{(8,351)}=3.53$ & 0.03 & $\chi_{(2)}^{2}=0.26$ & 0.88 \\
\hline
\end{tabular}

Table 3. Model comparison for Experiment 2, assessing the contribution of each variable (suppression at electrodes C3, C4, 01, 02; condition; and the interaction between them) to the goodness-of-fit of the (Left) linear mixed-effects models predicting participants' accuracy at rating the target's affect across each video, and the (Right) generalized linear mixedeffects model predicting whether a participant's rating change at the epoch level (increased, decreased, or maintained, compared to the previous epoch) matched the target's rating change. 
This model revealed again, as in Experiment 1, main effects of condition: higher empathic accuracy for the Audio-Video condition compared to the Video-Only condition ( $\beta$ $=1.27[1.07,1.46], t=12.54$, Bonferroni-corrected $p<0.001)$, and higher empathic accuracy in the Audio-Only condition compared to the Video-Only condition $(\beta=1.22[1.01$ ,1.42], $t=-11.72, p<0.001$ ), with no difference between the Audio-Video and Audio-Only conditions $(\beta=0.05[-0.14,0.24], t=0.52, p=1.00$; see Figure 5B). However, different from Experiment 1, we found a significant interaction between mu suppression at C4 and condition, such that greater mu suppression at C4 was associated with higher empathic accuracy only for the Video-Only condition compared to the Audio-Video condition $(\beta=-$ $0.25[-0.46,-0.05], t=-2.41, p=0.02$; see Figure 5C), and for the Video-Only condition compared to the Audio-Only condition $(\beta=-0.22[-0.41,-0.03], t=-2.31, p=0.02)$. The simple slope of mu suppression at C4 in the Video-Only condition when controlling for all the other variables was marginally significant $(\beta=-0.15[-0.30,-0.00], t=-1.94, p=0.053)$. No other variable was significantly correlated with empathic accuracy (see Table 4). All results remain the same when including only the 43 right-handed participants (for the analysis, see supplementary materials). In the supplementary, we also added the reanalysis of Experiment 1 with outlier removal. This analysis demonstrates a similar correlation trend between mu suppression and empathic accuracy though not significant. Furthermore, we re-analyzed the results of Experiment 2 without outlier removal. In this analysis, the correlation between mu suppression and empathic accuracy was not found to be significant (for the analysis, see supplementary materials). 


\begin{tabular}{|c|c|c|c|c|c|c|c|c|}
\hline \multirow[b]{2}{*}{ Predictors } & \multicolumn{4}{|c|}{$\begin{array}{l}\text { Video-Level Models: } \\
\text { Empathic Accuracy }\end{array}$} & \multicolumn{4}{|c|}{$\begin{array}{l}\text { Epoch-Level Models: } \\
\text { Change Detection }\end{array}$} \\
\hline & $\beta$ & $S E$ & $C I$ & $p$ & $\beta$ & $S E$ & $C I$ & $p$ \\
\hline Intercept & 0.38 & 0.13 & $0.12,0.64$ & $<0.001$ & -0.29 & 0.06 & $-0.41,-0.16$ & $<0.001$ \\
\hline C3 suppression & -0.04 & 0.05 & $-0.14,0.07$ & 0.48 & 0.01 & 0.02 & $-0.03,0.04$ & 0.63 \\
\hline $\begin{array}{l}\text { C4 suppression } \\
\text { (simple slope in } \\
\text { Audio-Video } \\
\text { condition) }\end{array}$ & 0.11 & 0.08 & $-0.06,0.27$ & 0.21 & -0.01 & 0.02 & $-0.04,0.03$ & 0.70 \\
\hline 01 suppression & -0.01 & 0.06 & $-0.12,0.11$ & 0.89 & -0.06 & 0.02 & $-0.10,-0.02$ & 0.002 \\
\hline 02 suppression & 0.03 & 0.06 & $-0.09,0.16$ & 0.58 & 0.03 & 0.02 & $-0.02,0.07$ & 0.22 \\
\hline $\begin{array}{l}\text { Video-Only vs. } \\
\text { Audio-Video }\end{array}$ & -1.27 & 0.10 & $-1.46,-1.07$ & $<0.001$ & -0.23 & 0.04 & $-0.30,-0.16$ & $<0.001$ \\
\hline $\begin{array}{l}\text { Audio-Only vs. } \\
\text { Audio-Video }\end{array}$ & -0.05 & 0.10 & $-0.24,0.14$ & 0.70 & 0.03 & 0.04 & $-0.04,0.10$ & 0.42 \\
\hline $\begin{array}{l}\text { C4 suppression* } \\
\text { Video-Only } \\
\text { interaction }\end{array}$ & -0.25 & 0.11 & $-0.46,-0.05$ & 0.017 & & & & \\
\hline $\begin{array}{l}\text { C4 suppression* } \\
\text { Audio-Video } \\
\text { interaction }\end{array}$ & -0.03 & 0.10 & $-0.23,0.16$ & 0.72 & & & & \\
\hline \multicolumn{9}{|l|}{$\begin{array}{l}\text { Random } \\
\text { Effects }\end{array}$} \\
\hline$\sigma^{2}$ & 0.05 & & & & 3.2 & & & \\
\hline \multirow[t]{2}{*}{$\tau_{00}$} & \multicolumn{4}{|c|}{0.00 participantID } & \multicolumn{4}{|c|}{0.01 participantID } \\
\hline & \multicolumn{4}{|c|}{$0.01_{\text {videoID }}$} & \multicolumn{4}{|c|}{0.03 videoID } \\
\hline ICC & \multicolumn{4}{|l|}{0.17} & \multicolumn{4}{|c|}{0.01} \\
\hline \multirow[t]{2}{*}{$\mathrm{N}$} & \multicolumn{4}{|c|}{46 participantID } & \multicolumn{4}{|c|}{46 participantID } \\
\hline & \multicolumn{4}{|c|}{9 videoID } & \multicolumn{4}{|c|}{$9_{\text {videoID }}$} \\
\hline Observations & \multicolumn{4}{|l|}{372} & \multicolumn{4}{|c|}{20890} \\
\hline $\begin{array}{l}\text { Marginal R2 / } \\
\text { Conditional R² }\end{array}$ & \multicolumn{4}{|c|}{$0.32 / 0.43$} & \multicolumn{4}{|c|}{$0.005 / 0.02$} \\
\hline
\end{tabular}



AIC
23.73
28120.66
Log-Likelihood
0.13
$-14051.33$

Table 4. Summary of statistical models for Experiment 2. Left: Results from a linear mixed-effects model predicting participants' accuracy at rating the target's affect across each video. Right: Results from a generalized linear mixed-effects model predicting whether a participant's rating change at the epoch level (increased, decreased, or maintained, compared to the previous epoch) matched the target's rating change. See Methods for operationalization of the dependent variables. $\beta$ indicates the standardized beta coefficients on suppression at the electrodes, where negative values indicate greater suppression. SE: standard error of the regressor. CI: confidence intervals of the standardized beta coefficients of the regressor.

\section{A}

Alpha and mu rhythms in the different conditions

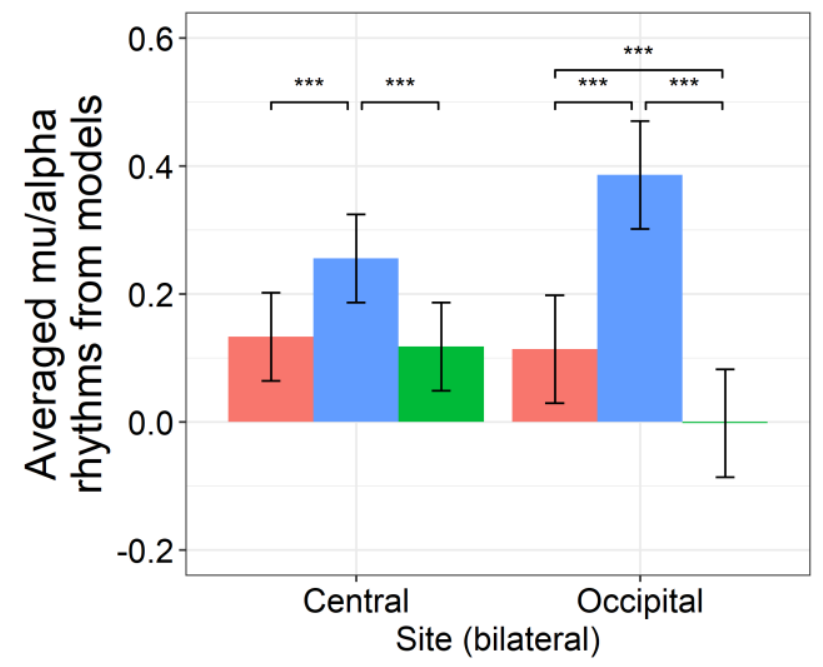

C

\section{Correlation between mu across conditions and empathic accuracy}

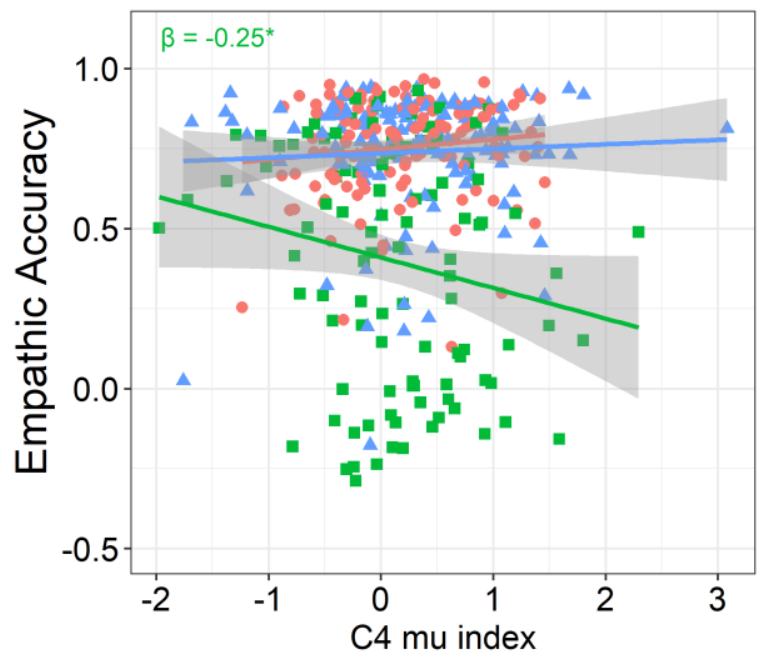

\section{Empathic Accuracy}

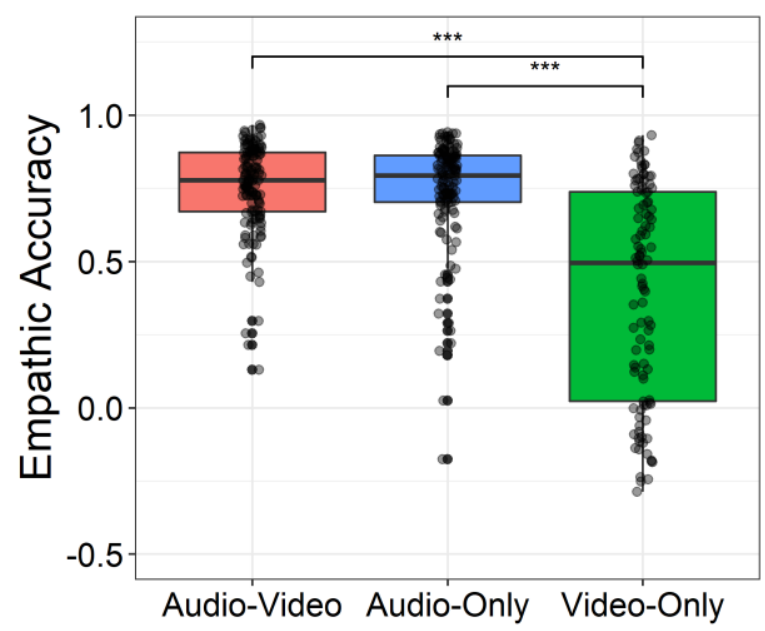

Condition

Audio-Video

Audio-Only

Video-Only 
Figure 5. Experiment 2 Results. (A) A barplot of mu and alpha rhythms in the different conditions over the central and occipital sites. Values are obtained from the linear mixed-effects models predicting participants' mu and alpha rhythms in the different conditions. More negative values indicate more suppression, relative to baseline. The error bars reflect SE.

(B) A boxplot of empathic accuracy in the different conditions. Values are obtained from the linear mixed-effects model predicting participants' accuracy at rating a target's affect across each video. (C) A scatterplot of empathic accuracy by viewing condition against averaged mu suppression in electrode C4. Data-points are colored by viewing condition. On the horizontal axis, more negative values indicate greater suppression. The line represents a best-fit line, showing a negative correlation between mu suppression and accuracy.

${ }^{*} p<.05,{ }^{* *} p<.01,{ }^{* * *} p<.001$

Finally, we turned to the epoch-level analysis. Model comparisons suggested that there were no significant interactions of suppression with condition, so in Table 4 we report the model without interactions. When considering the main effects of condition, similar to the video-level model, this model revealed enhanced change detection for the Audio-Video condition compared to the Video-Only condition $(\beta=0.23[016,0.30], t=6.64$, Bonferroni-corrected $p<0.001)$. Enhanced change detection was also found in the AudioOnly condition compared to the Video-Only condition $(\beta=0.26[0.19,0.33], t=-7.24, p<$ 0.001). No difference was found in change detection between Audio-Video and Audio-Only conditions $(\beta=-0.03[-0.10,0.04], t=-0.81, p=1.00)$. This model also indicated that greater alpha suppression in 01 (i.e., less activation of alpha rhythms over the left occipital cortex) was significantly associated with enhanced change detection $(\beta=-0.06[-0.10$, 0.02], $t=-3.03, p=0.002$ ). No significant correlation was found between alpha suppression in 02 and change detection. Furthermore, no significant correlation was found between mu suppression in C3 and C4 and change detection (see Table 4).

Note, that following this analysis, we re-analyzed the data from Experiment 1, extracting individualized $2 \mathrm{~Hz}$ frequency bands of mu rhythm for each participant. The 
results are similar but not identical, and the correlation between mu suppression and empathic accuracy is marginally significant $(p=0.119)$, as well as for the correlation between mu suppression enhanced change detection $(p=0.064$, for the analysis, see supplementary materials).

Lastly, we investigated whether the change detection score and empathic accuracy scores were correlated, and revealed that there is indeed a weak but significant correlation between them in both studies, strengthening the notion that the two measures are related, yet not identical, and enable capturing different aspects of accuracy (see supplementary materials for the full analyses

\section{General Discussion}

Our results are the first to demonstrate that mu suppression tracks accurate emotion judgments in more complex, naturalistic settings, at least when there is only visual information to rely on. This strengthens the role of sensorimotor representations in social cognition, in making social inferences from sensorimotor cues such as facial expressions or body motion (e.g., Moore et al., 2012; Perry, Troje, et al., 2010). In Experiment 1, although we found differences in mean levels of mu suppression across the three conditions, we observed that mu suppression was related to empathic accuracy across all conditions, as hypothesized. This suggests that even in the absence of facial expressions (i.e., in the AudioOnly condition), sensorimotor regions were still engaged to produce representations that contribute to accurate empathic judgments. This relates to previous work reporting mirror-neuron sensitivity to auditory cues, both in monkeys and in humans (see Gazzola et 
al., 2006; Kohler et al., 2002, for evidence from humans and monkeys, respectively). There are several, not mutually exclusive, possible levels of the stimuli that the mu signature may be tracking in the auditory task: First, mu suppression has been shown to be sensitive to concrete action sentences (Moreno et al., 2015) and may be sensitive to actions (including emotional physical reactions) described by the targets. Second, it may be sensitive to lowlevel paralinguistics cues (e.g., changes in tone or pitch), which could in turn support higher-level empathic inferences. These options could be further investigated in future research.

Importantly though, these results did not fully replicate in Experiment 2. In this experiment, the correlation of empathic accuracy to mu suppression across the three conditions was marginally significant $(p=.053)$ only when narrowing our focus to the more sensitive individualized mu frequency bands. We additionally found a significant interaction between mu suppression and the Visual-Only condition. There could be several explanations for these discrepancies:

Our experiments, as well as those of others (Gesn \& Ickes, 1999; Hall \& Schmid Mast, 2007; Jospe et al., 2020; Kraus, 2017), demonstrate that empathic-accuracy abilities are primarily dependent on the narrative that comes from the auditory information. It is therefore likely that when the narrative is present, empathic accuracy relies more on mentalizing, and thus on other brain regions (such as the vmPFC or the TPJ; see Atique et al., 2011; Van Overwalle \& Baetens, 2009, for reviews). However, in the absence of a narrative, as in the Video-Only condition, the more dominant mechanism may be sensorimotor simulation, which may explain why we found (in Experiment 2) the greater correlation between mu suppression and empathic accuracy in the Video-Only condition. 
The second explanation is the difference in the stimuli used. Indeed, in the second experiment empathic accuracy has much less variance in the auditory conditions, but not in the Video-Only condition. There is less chance of finding any correlation with less variance, which could explain the interaction found between mu suppression and the Video-Only condition. Note that although the average targets' emotional intensity ratings are mostly similar across the two different experiments (see supplementary tables 1 and 2), the emotional intensity of the mostly positive clips in experiment 2 is lower than those of experiment 1 , which may contribute to the result differences between the two experiments. The influence of the target emotional valence and intensity, the target and participant gender, as well as other participants' characteristics (e.g., age, ethnicity, empathy trait) on the correlation between mu suppression and empathic accuracy, should be tested in future studies, either with larger sample sizes or in an experimental design adapted specifically for answering these important questions.

Note that experiment 1 was conducted on a very diverse US sample with varied ethnic backgrounds and experiment 2 on an Israeli sample. As the samples' diversity could potentially moderate the correlation between mu suppression and empathic accuracy, it strengthens the robustness of the findings beyond different races and ethnicity and may even play a role in the discrepancies between the two experiments' findings. Future studies should investigate if ethnicity, race or other intergroup differences influence the relationship between mu suppression and empathic accuracy. Moreover, as the two samples have a different proportion of females and males, future studies should investigate if gender affects the relationship between mu suppression and empathic accuracy. 
The third explanation is the lower power in Experiment 1, which makes it harder to reveal a significant interaction between mu suppression and condition. This suggest that mu suppression may indeed be more strongly related to empathic processes through the visual domain.

The fourth explanation is of course that our results from Experiment 2 represent a more accurate description of the world. Even if this is the case, we now show that in two EEG experiments, using naturalistic stimuli, and across languages and cultures, mu suppression-a proxy for sensorimotor simulation-contributes to accurate empathic judgments. This simulation may be more evident when there is only visual information to rely on. The differences and similarities between the first and second experiments stress the importance of replication and larger samples in EEG studies.

Our findings add to a small but growing set of studies suggesting that both experience sharing and mentalizing systems contribute to making complex, naturalistic judgments. While the current study stresses the role of sensorimotor activation, presumably supporting experience sharing, there is other evidence that both experience sharing and mentalizing are important. In an earlier fMRI study (Zaki et al., 2009b), accurate empathic judgments engaged mentalizing regions like the medial prefrontal cortex, as well as regions thought to support experience sharing such as the premotor cortex. One important difference we note is that in Zaki and colleagues' (2009b) fMRI study, fMRI and behavioral ratings were collected simultaneously the first and only time the participants saw the videos. In the present study, participants saw all videos twice, the first time with EEG, and the second time for collecting their behavioral rating. This was done in order to avoid contamination of the EEG mu suppression signal, which is highly 
sensitive to motor movement. This limitation forced us to use this non-ideal experimental design which had the potential to conceal the role of mu suppression in empathic processes. This, in fact, provides a stronger, more conservative test of the robustness of these sensorimotor representations, as even in this case, EEG mu suppression from the first viewing was correlated with ratings made during the second viewing.

Finally, though we did not predict lateralization, our finding that empathic accuracy is related to mu suppression only over the right sensorimotor cortex corroborates previous studies that found right-lateralized mu suppression in perceiving emotional expressions, both in adults (Moore et al., 2012) and in children (Rayson et al., 2016). This speaks to a larger consensus in the literature (see Adolphs, 2002, for review) that finds righthemisphere functionalization of emotion recognition from facial expressions (e.g., Killgore \& Yurgelun-Tfodd, 2007) as well as prosody (e.g., Adolphs et al., 2002).

This study has some limitations. First, our second experiment, albeit having a larger sample size, had less variability in some of the behavioral measures and replicated only some of the initial findings from Experiment 1. Second, as mentioned above, to minimize contamination of the EEG data by motor movement, participants saw all videos twice, and the behavioral ratings were recorded only during the second time they saw the video (without EEG), which may have led to biases in their ratings.

To conclude, the current study reveals an EEG measure of sensorimotor representations, indexed by mu rhythm suppression, that contributes to the accuracy of complex naturalistic empathic judgments. 


\section{Acknowledgments}

We would like to acknowledge Hailey Gordon and Atiriya Hari for help with recruiting and running participants, and Valerie Taylor and Kira Alqueza for help with collecting the stimuli. This work was supported in part by a US-Israel Binational Science Foundation grant to JZ and AP, a fellowship from the Azrieli Foundation to AP, a NIH Grant 1R01MH112560-01 to JZ, a Stanford IRiSS Computational Social Science Fellowship, and a Singapore Ministry of Education Academic Research Fund Tier 1 grant to DCO.

\section{References}

Adolphs, R. (2002). Neural systems for recognizing emotion. Current Opinion in Neurobiology, 12(2), 169-177. https://doi.org/10.1016/S0959-4388(02)00301-X

Adolphs, R., Damasio, H., \& Tranel, D. (2002). Neural Systems for Recognition of Emotional Prosody. A 3-D Lesion Study. Emotion, 2(1), 23-51. https://doi.org/10.1037/15283542.2 .1 .23

Aguinis, H., Gottfredson, R. K., \& Culpepper, S. A. (2013). Best-Practice Recommendations for Estimating Cross-Level Interaction Effects Using Multilevel Modeling. In Journal of Management (Vol. 39, Issue 6). https://doi.org/10.1177/0149206313478188

Atique, B., Erb, M., Gharabaghi, A., Grodd, W., \& Anders, S. (2011). Task-specific activity and connectivity within the mentalizing network during emotion and intention mentalizing. NeuroImage, 55(4), 1899-1911. https://doi.org/10.1016/j.neuroimage.2010.12.036 
Bates, D., Mächler, M., Bolker, B. M., \& Walker, S. C. (2015). Fitting linear mixed-effects models using lme4. Journal of Statistical Software, 67(1), 1-48. https://doi.org/10.18637/jss.v067.i01

Chiang, A. K. I., Rennie, C. J., Robinson, P. A., van Albada, S. J., \& Kerr, C. C. (2011). Age trends and sex differences of alpha rhythms including split alpha peaks. Clinical Neurophysiology, 122(8), 1505-1517. https://doi.org/10.1016/j.clinph.2011.01.040

Cohen, M. X. (2021). A data-driven method to identify frequency boundaries in multichannel electrophysiology data. Journal of Neuroscience Methods, 347(July 2020), 108949. https://doi.org/10.1016/j.jneumeth.2020.108949

Cuellar, M., Bowers, A., Harkrider, A. W., Wilson, M., \& Saltuklaroglu, T. (2012). Mu suppression as an index of sensorimotor contributions to speech processing: Evidence from continuous EEG signals. International Journal of Psychophysiology, 85(2), 242248. https://doi.org/10.1016/j.ijpsycho.2012.04.003

Decety, J., \& Meyer, M. (2008). From emotion resonance to empathic understanding: A social developmental neuroscience account. Development and Psychopathology, 20(4), 1053-1080. https://doi.org/10.1017/S0954579408000503

Devlin, H. C., Zaki, J., Ong, D. C., \& Gruber, J. (2016). Tracking the Emotional Highs but Missing the Lows: Hypomania Risk is Associated With Positively Biased Empathic Inference. Cognitive Therapy and Research, 40(1), 72-79. https://doi.org/10.1007/s10608-015-9720-6

DiGirolamo, M. A., Simon, J. C., Hubley, K. M., Kopulsky, A., \& Gutsell, J. N. (2019). Clarifying the relationship between trait empathy and action-based resonance indexed by EEG mu-rhythm suppression. Neuropsychologia, 133(August), 107172. 
https://doi.org/10.1016/j.neuropsychologia.2019.107172

Ensenberg, N. S., Perry, A., \& Aviezer, H. (2017). Are you looking at me? Mu suppression modulation by facial expression direction. Cognitive, Affective and Behavioral Neuroscience, 17(1), 174-184. https://doi.org/10.3758/s13415-016-0470-z

Eyal, T., Steffel, M., \& Epley, N. (2018). Perspective mistaking: Accurately understanding the mind of another requires getting perspective, not taking perspective. Journal of Personality and Social Psychology, 114(4), 547-571. https://doi.org/10.1037/pspa0000115

Flanagin, A., Frey, T., \& Christiansen, S. L. (2021). Updated Guidance on the Reporting of Race and Ethnicity in Medical and Science Journals. JAMA - Journal of the American Medical Association, 326(7), 621-627. https://doi.org/10.1001/jama.2021.13304

Fox, N. A., Bakermans-Kranenburg, M. J., Yoo, K. H., Bowman, L. C., Cannon, E. N., Vanderwert, R. E., \& ... \& Van IJzendoorn, M. H. (2016). Assessing Human Mirror Activity with EEG Mu Rhythm : A Meta-Analysis. 142(3), 291-313.

Gazzola, V., Aziz-Zadeh, L., \& Keysers, C. (2006). Empathy and the Somatotopic Auditory Mirror System in Humans. Current Biology, 16(18), 1824-1829. https://doi.org/10.1016/j.cub.2006.07.072

Gesn, P. R., \& Ickes, W. (1999). The development of meaning contexts for empathic accuracy: Channel and sequence effects. Journal of Personality and Social Psychology, 77(4), 746-761. https://doi.org/10.1037/0022-3514.77.4.746

Gutsell, J. N., Simon, J. C., \& Jiang, Y. (2020). Perspective taking reduces group biases in sensorimotor resonance. Cortex, 131, 42-53. https://doi.org/10.1016/j.cortex.2020.04.037 
Halekoh, U., \& Højsgaard, S. (2014). A kenward-Roger approximation and parametric bootstrap methods for tests in linear mixed models-the R package pbkrtest. Journal of Statistical Software, 59(9), 1-32. https://doi.org/10.18637/jss.v059.i09

Hall, J. A., \& Schmid Mast, M. (2007). Sources of accuracy in the empathic accuracy paradigm. Emotion, 7(2), 438-446. https://doi.org/10.1037/1528-3542.7.2.438

Hobson, H. M., \& Bishop, D. V. M. (2017). The interpretation of mu suppression as an index of mirror neuron activity: Past, present and future. Royal Society Open Science, 4(3). https://doi.org/10.1098/rsos.160662

Jenson, D., Bowers, A. L., Harkrider, A. W., Thornton, D., Cuellar, M., \& Saltuklaroglu, T. (2014). Temporal dynamics of sensorimotor integration in speech perception and production: Independent component analysis of EEG data. Frontiers in Psychology, 5(JUL), 1-17. https://doi.org/10.3389/fpsyg.2014.00656

Jospe, K., Genzer, S., Klein Selle, N., Ong, D., Zaki, J., \& Perry, A. (2020). The contribution of linguistic and visual cues to physiological synchrony and empathic accuracy. Cortex.

Jung, T. P., Makeig, S., Westerfield, M., Townsend, J., Courchesne, E., \& Sejnowski, T. J. (2000). Removal of eye activity artifacts from visual event-related potentials in normal and clinical subjects. Clinical Neurophysiology, 111(10), 1745-1758.

https://doi.org/10.1016/S1388-2457(00)00386-2

Keysers, C., Kaas, J. H., \& Gazzola, V. (2010). Somatosensation in social perception. Nature Reviews Neuroscience, 11(6), 417-428. https://doi.org/10.1038/nrn2833

Killgore, W. D. S., \& Yurgelun-Todd, D. A. (2007). The right-hemisphere and valence hypotheses: Could they both be right (and sometimes left)? Social Cognitive and Affective Neuroscience, 2(3), 240-250. https://doi.org/10.1093/scan/nsm020 
Klimesch, W. (2012). Alpha-band oscillations, attention, and controlled access to stored information. Trends in Cognitive Sciences, 16(12), 606-617. https://doi.org/10.1016/j.tics.2012.10.007

Kohler, E., Keysers, C., Umilta, M. A., Fogassi, L., Gallese, V., \& Rizzolatti, G. (2002). Hearing sounds, understanding actions: Action representation in mirror neurons. Science, 297(5582), 846-848. https://doi.org/10.1126/science.1070311

Kraus, M. W. (2017). Voice-only communication enhances empathic accuracy. American Psychologist, 72(7), 644-654. https://doi.org/10.1037/amp0000147

Le Bel, R. M., Pineda, J. A., \& Sharma, A. (2009). Motor-auditory-visual integration: The role of the human mirror neuron system in communication and communication disorders. Journal of Communication Disorders, 42(4), 299-304. https://doi.org/10.1016/j.jcomdis.2009.03.011

Lenth, R., Buerkner, P., Herve, M., LOve, J., Riebl, H., \& Singmann, H. (2020). emmeans: Estimated Marginal Means, aka Least-Squares Means. https://cran.rproject.org/web/packages/emmeans/index.html

Lepage, J. F., \& Théoret, H. (2006). EEG evidence for the presence of an action observationexecution matching system in children. European Journal of Neuroscience, 23(9), 25052510. https://doi.org/10.1111/j.1460-9568.2006.04769.x

Levenson, R. W., \& Ruef, A. M. (1992). Empathy: A Physiological Substrate. Journal of Personality and Social Psychology, 63(2), 234-246. https://doi.org/10.1037/00223514.63.2.234

Lüdecke, D. (2021). Data Visualization for Statistics in Social Science [R package sjPlot version 2.8.7]. https://cran.r-project.org/package=sjPlot 
Moore, A., Gorodnitsky, I., \& Pineda, J. A. (2012). EEG mu component responses to viewing emotional faces. Behavioural Brain Research, 226(1), 309-316. https://doi.org/10.1016/j.bbr.2011.07.048

Moreno, I., de Vega, M., León, I., Bastiaansen, M., Glen Lewis, A., \& Magyari, L. (2015). Brain dynamics in the comprehension of action-related language. A time-frequency analysis of mu rhythms. NeuroImage, 109, 50-62. https://doi.org/10.1016/j.neuroimage.2015.01.018

Muthukumaraswamy, S. D., \& Johnson, B. W. (2004). Changes in rolandic mu rhythm during observation of a precision grip. Psychophysiology, 41(1), 152-156. https://doi.org/10.1046/j.1469-8986.2003.00129.x

Nakagawa, S., \& Schielzeth, H. (2013). A general and simple method for obtaining R2 from generalized linear mixed-effects models. Methods in Ecology and Evolution, 4(2), 133142. https://doi.org/10.1111/j.2041-210x.2012.00261.x

Ong, D. C., Wu, Z., Zhi-Xuan, T., Reddan, M., Kahhale, I., Mattek, A., \& Zaki, J. (2019). Modeling emotion in complex stories: the Stanford Emotional Narratives Dataset. IEEE Transactions on Affective Computing.

Ong, D. C., Zaki, J., \& Goodman, N. D. (2015). Affective cognition: Exploring lay theories of emotion. Cognition, 143, 141-162. https://doi.org/10.1016/j.cognition.2015.06.010

Perry, A., \& Bentin, S. (2009). Mirror activity in the human brain while observing hand movements: A comparison between EEG desynchronization in the $\mu$-range and previous fMRI results. Brain Research, 1282, 126-132. https://doi.org/10.1016/j.brainres.2009.05.059

Perry, A., Bentin, S., Bartal, I. B. A., Lamm, C., \& Decety, J. (2010). “Feeling” the pain of those 
who are different from us: Modulation of EEG in the mu/alpha range. Cognitive, Affective and Behavioral Neuroscience, 10(4), 493-504.

https://doi.org/10.3758/CABN.10.4.493

Perry, A., Stein, L., \& Bentin, S. (2011). Motor and attentional mechanisms involved in social interaction-Evidence from mu and alpha EEG suppression. NeuroImage, 58(3), 895904. https://doi.org/10.1016/j.neuroimage.2011.06.060

Perry, A., Troje, N. F., \& Bentin, S. (2010). Exploring motor system contributions to the perception of social information: Evidence from EEG activity in the mu/alpha frequency range. Social Neuroscience, 5(3), 272-284. https://doi.org/10.1080/17470910903395767

Pineda, J. A. (2005). The functional significance of mu rhythms: Translating "seeing" and "hearing" into "doing." Brain Research Reviews, 50(1), 57-68. https://doi.org/10.1016/j.brainresrev.2005.04.005

Pineda, J. A., \& Hecht, E. (2009). Mirroring and mu rhythm involvement in social cognition: Are there dissociable subcomponents of theory of mind? Biological Psychology, 80(3), 306-314. https://doi.org/10.1016/j.biopsycho.2008.11.003

Popov, T., Miller, G. A., Rockstroh, B., \& Weisz, N. (2013). Modulation of $\alpha$ power and functional connectivity during facial affect recognition. Journal of Neuroscience, 33(14), 6018-6026. https://doi.org/10.1523/JNEUROSCI.2763-12.2013

R Core Team. (2020). R: The R Project for Statistical Computing. https://www.r-project.org/ Rayson, H., Bonaiuto, J. J., Ferrari, P. F., \& Murray, L. (2016). Mu desynchronization during observation and execution of facial expressions in 30-month-old children. Developmental Cognitive Neuroscience, 19, 279-287. 
https://doi.org/10.1016/j.dcn.2016.05.003

Saxe, R., \& Houlihan, S. D. (2017). Formalizing emotion concepts within a Bayesian model of theory of mind. Current Opinion in Psychology, 17, 15-21. https://doi.org/10.1016/j.copsyc.2017.04.019

Simon, J. C., \& Gutsell, J. N. (2021). Recognizing humanity: dehumanization predicts neural mirroring and empathic accuracy in face-to-face interactions. Social Cognitive and Affective Neuroscience, June 2020, 463-473. https://doi.org/10.1093/scan/nsab014

Singer, T., Seymour, B., O’Doherty, J., Kaube, H., Dolan, R. J., \& Frith, C. D. (2004). Empathy for Pain Involves the Affective but not Sensory Components of Pain. Science, 303(5661), 1157-1162. https://doi.org/10.1126/science.1093535

Skerry, A. E., \& Saxe, R. (2015). Neural Representations of Emotion Are Organized around Abstract Event Features. Current Biology, 25(15), 1945-1954. https://doi.org/10.1016/j.cub.2015.06.009

Van Overwalle, F., \& Baetens, K. (2009). Understanding others' actions and goals by mirror and mentalizing systems: A meta-analysis. NeuroImage, 48(3), 564-584. https://doi.org/10.1016/j.neuroimage.2009.06.009

Zaki, J. (2014). Empathy: A motivated account. Psychological Bulletin, 140(6), 1608-1647. http://psycnet.apa.org/journals/bul/140/6/1608/

Zaki, J., Bolger, N., \& Ochsner, K. (2008). It takes two: The interpersonal nature of empathic accuracy. Psychological Science, 19(4), 399-404.

Zaki, J., Bolger, N., \& Ochsner, K. (2009). Unpacking the Informational Bases of Empathic Accuracy. Emotion, 9(4), 478-487. https://doi.org/10.1037/a0016551

Zaki, J., \& Ochsner, K. (2011). Reintegrating the study of accuracy into social cognition 
research. Psychological Inquiry, 22(3), 159-182.

https://doi.org/10.1080/1047840X.2011.551743

Zaki, J., \& Ochsner, K. (2012). The neuroscience of empathy: Progress, pitfalls and promise. Nature Neuroscience, 15(5), 675-680. https://doi.org/10.1038/nn.3085

Zaki, J., Weber, J., Bolger, N., \& Ochsner, K. (2009). The neural bases of empathic accuracy. Proceedings of the National Academy of Sciences of the United States of America, 106(27), 11382-11387. https://doi.org/10.1073/pnas.0902666106 